\title{
A study on bentonite and kenaf properties for grounding purposes
}

\begin{abstract}
There are many methods that can be used in order to increase the performance of a grounding system meant for power system protection, i.e. by lowering its earth resistance values, which one of them is where soil could be treated using chemical or natural enhancement materials. This technique is one of the effective ways to improve the performance of a grounding system in which this work employed $15 \mathrm{~kg}$ Bentonite, and $3 \mathrm{~kg}$ Kenaf in powder and fibre forms as the natural enhancement materials for grounding systems at which they were either mixed or layered. The main purpose of conduction this work is to compare the performance of natural enhancement material mixture grounding systems to a Reference grounding system, which is without any enhancement material in the vicinity of the ground conductor. Fall-of-potential method was employed to measure earth resistance for the performances of the four grounding systems to be compared. After 60 days, their performances in descending order were Bentonite and Kenaf powder layered, Bentonite and Kenaf powder mixed, Bentonite and Kenaf fibre layered and Reference grounding systems. Hence, the grounding system with Bentonite and Kenaf powder layered is proposed to be the best to be used in order to reduce the earth resistance as it has the lowest earth resistance and able to stabilize faster compared to the other three grounding systems.
\end{abstract}

Keyword: Grounding system; Bentonite; Kenaf; Natural enhancement material 